Dhaka Univ. J. Sci. 61(1): 145-148, 2013 (January)

\title{
Chitosan and Carboxymethyl Chitosan from Fish Scales of Labeo rohita
} Tanvir Muslim*, Mohammad Habibur Rahman, Hosne Ara Begum and Md. Azizur Rahman

\author{
Department of Chemistry, Dhaka University, Dhaka-1000, Bangladesh
}

Received on 10.09.2012. Accepted for Publication on 13. 10. 2012

\begin{abstract}
Chitin was extracted from the fish scales of Labeo rohita and chitosan was successfully prepared from it by deacetylation reaction. The prepared chtiosan was characterized by FT-IR spectral analysis and degree of decetylation was determined by pH-metric titration. The molecular weight of chitosan was estimated by viscometric method. Chitosan was converted into its carboxymethyl derivative using alkali and monochloroacetic acid. The prepared carboxymethyl chitosan was characterized by FT-IR spectral analysis and degree of substitution was estimated.
\end{abstract}

Keywords: Fish scales, Labeo rohita, chitosan, carboxymethyl chitosan, characterisation.

\section{Introduction}

Chitin is a natural polysaccharide, poly- $[\beta-(1 \rightarrow 4)-2-$ acetamido-2-deoxy-glucose] (Fig. 1), found particularly in the shells of crustaceans such as crab and shrimp, the cuticles of insects, and the cell walls of fungi ${ }^{1}$. It is the second most abundant polysaccharide in nature, after cellulose. Chitosan is the $N$-deacetylated derivative of chitin, prepared by $N$-deacetylation with alkali solution at elevated temperature (Fig. 1). Chitosan naturally exists only in some species of fungi. A sharp nomenclature with respect to the degree of $N$-deacetylation has not been defined between chitin and chitosan ${ }^{2,3}$. From a practical viewpoint, shells of crustaceans such as crabs and shrimps are conveniently available as wastes from seafood processing industries and are used for the commercial production of chitin. As functional materials, chitin and chitosan offer a unique set of characteristics: biocompatibility, biodegradability, nontoxicity, physiological inertness, immunological activity, antibacterial properties, wound-healing activity, heavy metal ions chelation, gel forming properties and hydrophilicity, and remarkable affinity to proteins. Therefore, chitin and chitosan have prospective applications in many fields such as wastewater treatment, food industry, biotechnology, agriculture, medical and pharmaceutical, cosmetics, and pulp-paper industries ${ }^{\mathbf{1 , 4}}$. Numerous research studies have been undertaken to prepare chitin and chitosan derivatives with well-defined structures by controlled chemical modification reactions and thereby to construct sophisticated molecular architecture having various advanced functions ${ }^{5,6}$. Chemical modification of chitosan arylaldehyde is a popular method for preparing new chitosan hybrids. Different chitosan hybrids were synthesised to determine the metal adsorption capacity ${ }^{7}$. Chitosan modified by poly(ethylene glycol) created attraction among the researchers to obtain hydrophilic derivatives and explore their new applications. The bioactivity of poly(ethylene glycol)-chitosan hybrids has been studied ${ }^{\mathbf{8}}$. Preparation, characterisation and applications of carboxymethyl chitosan for the controlled release of drugs, orthopedic devices and tissue adhesion have also been reported ${ }^{9,10}$.

Although Bangladesh is rich in sources of chitin and chitosan, applications of these natural polymers are very limited. Still chitosan is not produced in this country on commercial basis. Some traders are exporting raw materials for production of chitin/chitosan to foreign countries. Bangladesh is lag behind in chitin/chitosan production and its utilization. So, study of preparation and application of chitosan and its derivatives is necessary. For, this purpose, low cost and available waste materials may be considered as sources of chitin. "Fish scales" are good source of chitin and chitosan. The fish scales are discarded daily as waste materials from fish markets, canteens, fish processing industries or kitchens. This abundant waste may pose environmental hazard due to the easy deterioration. The use of this waste to produce valuable and biologically sustainable materials is a challenge for current research and development ${ }^{11}$. Generally, fish scales consist of protein (type I collagen and ichthylepidin) and apatite (calcium phosphate, magnesium carbonate and calcium carbonate) ${ }^{\mathbf{1 2}}$. A very few information is available related to chitosan from fish scales. A study on dye-binding interaction of chitosan obtained from the fish scale of Tilapia (Tilapia nilotica) was reported by Uawonggul et.al ${ }^{13}$. Recently, a report on extraction and characterization of chitin from scales of common carp fish (Cyprinus carpio 1.) has been published ${ }^{\mathbf{1 1}}$.

The aim of present study is based on evaluation of the status of chitosan from fish scales of Labeo rohita (locally known as Rui of the carp family Cyprinidae), a common waste material, and preparation of carboxymethyl derivative from it.

\footnotetext{
*Corresponding author
} 


\section{Experimental}

All chemicals and solvents used in the present work were analytical grade (Merck and BDH). All solvents were distilled before use. Different solutions and reagents were prepared using analytical procedure.

\section{Collection and preparation of samples}

The raw fish scales of Labeo rohita was collected from a local market of Dhaka City, Bangladesh. The raw fish scales were washed with water and dried in sun for 3 days. After drying, the dried materials were ground into small pieces and kept in air tight container.

\section{Isolation of chitin from fish scales of Labeo rohita}

Chitin was prepared from the dried fish scales of Labeo rohita as described previously ${ }^{14}$ with minor modification. The process mainly involved the following steps-

(i) Demineralisation of fish scales: The dried fish scales was demineralised with hydrochloric acid solution (1.0 M) at room temperature with constant stirring for 2 hours, using a ratio of solid to acidic solution of $1: 13(\mathrm{w} / \mathrm{v})$, when the fish scales became quite squashy. Then those were rinsed with distilled water to remove acid and salt. The decalcified product was washed with methanol and acetone. The washed material was transferred to glass tray, and dried for overnight at $60^{\circ} \mathrm{C}$ in an oven.

(ii) Deproteinisation of fish scales: Deproteinisation was carried out by slowly adding the demineralised fish scales to sodium hydroxide solution $(1.0 \mathrm{M})$ to obtain a ratio of solid to alkaline solution of $1: 13(\mathrm{w} / \mathrm{v})$. The temperature of the reaction mixture was maintained at $60^{\circ} \mathrm{C}$ with constant stirring for 5 hours. The residue was then collected and washed with distilled water until the $\mathrm{pH}$ became neutral. Then it was washed with organic solvents and dried, as described above. The final product was obtained as chitin.

\section{Preparation of chitosan from isolated chitin}

Chitosan was prepared from isolated chitin as described previously ${ }^{14}$ with minor modification (Fig. 1). Isolated chitin was slowly added into a flask containing a solution of sodium hydroxide $(40 \%, \mathrm{w} / \mathrm{v})$ to obtain a ratio of solid to alkaline solution of $1: 15(\mathrm{w} / \mathrm{v})$. The temperature of reaction mixture was maintained at $100^{\circ} \mathrm{C}$ and refluxed under nitrogen atmosphere for 8 hours to remove some or all of the acetyl groups from amino groups on the polymer. The prepared chitosan was dissolved in acetic acid solution $(5 \%, \mathrm{w} / \mathrm{v})$ to obtain a ratio of solid to acidic solution of $1: 10(\mathrm{w} / \mathrm{v})$ and it was stirred continuously for two hours. The solution was kept over night and centrifuged. The clear supernatant liquid was taken in a beaker and sodium hydroxide solution $(5 \%, \mathrm{w} / \mathrm{v})$ was added drop wise into the acidic chitosan solution to obtain the precipitate of purified chitosan. The precipitate was thoroughly washed with distilled until the $\mathrm{pH}$ became neutral. Finally the purified chitosan was washed with organic solvents and dried in a similar way it was done for the chitin sample.
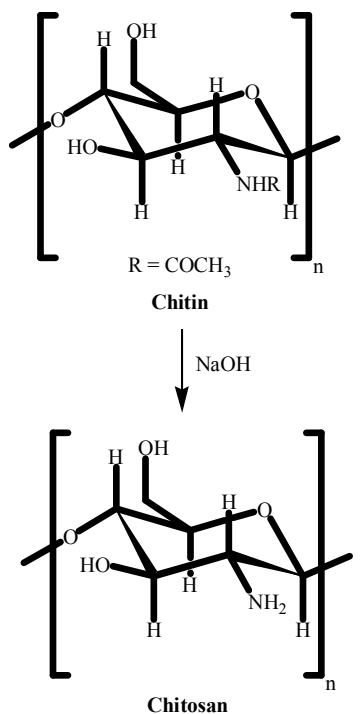

Fig. 1. Preparation of chitosan from chitin by deacetylation

\section{Characterization of chitosan}

FTIR sptectrum of prepared chitosan sample was recorded using a FTIR spectrophotometer (FT-IR 8400S, Shimadzu, Japan) within the range of $400-4000 \mathrm{~cm}^{-1}$ as $\mathrm{KBr}$ disc.

Degree of deacetylation (DDA) of prepared chitosan was determined by $\mathrm{pH}$-metric titration ${ }^{9}$. $\mathrm{pH}$ vs. volume of sodium hydroxide titration curves (smoothing $1^{\text {st }}$ derivative $\& 2^{\text {nd }}$ derivative) were prepared and analyzed by using software CurTipot $(\mathrm{pH} \&$ acid base titration curve: Analysis \& simulation) version 3.3.1 (2008) for MS Excel ${ }^{15}$.

Molecular weight of prepared chitosan was determined by viscometric method ${ }^{1,16,17}$. For the determination of viscosity average molecular weight $\left(\mathrm{M}_{\mathrm{v}}\right)$, the chitosan was dissolved in $0.1 \mathrm{M}$ acetic acid with $0.2 \mathrm{M} \mathrm{NaCl}(1: 1, \mathrm{v} / \mathrm{v})$. Ubbelohde capillary viscometer in a constant temperature water bath at $25^{\circ} \mathrm{C}$ was used to measure the intrinsic viscosity [ $\left.\boldsymbol{\eta}\right]$. The Mark-Houwink equation relating to intrinsic viscosity with 
empirical viscometric constants ${ }^{1} \mathrm{~K}=1.81 \times 10^{-3} \mathrm{~cm}^{3} / \mathrm{g}$ and $\mathrm{a}=0.93$ for chitosan was used to calculate the molecular weight using this equation: $[\boldsymbol{\eta}]=\mathrm{KM}_{\mathrm{V}}^{\mathrm{a}}$.
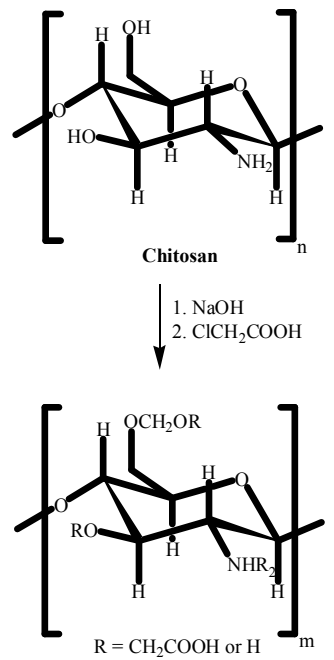

Carboxymethyl chitosa

Fig. 2. Preparation of carboxymethyl chitosan

Preparation of carboxymethyl chitosan from prepared chitosan

Carboxymethyl derivative of the prepared chitosan was carried out according to the method described previourly ${ }^{9}$ with minor modification (Fig. 2). Purified chitosan (1.5 g) was dispersed in iso-propanol $(33 \mathrm{~mL})$. After 20 minutes of magnetic stirring at room temperature, aqueous $\mathrm{NaOH}$ $(40 \%, 5.5 \mathrm{~g})$ and monochloroacetic acid/iso-propanol solution $(1: 1 \mathrm{~m} / \mathrm{m}, 19.2 \mathrm{~g})$ were added to the suspension. The reaction proceeded to the desired time ( 24 hours) at room temperature and the solid product was then filtered, suspended in $150 \mathrm{~mL}$ of methanol and neutralized with glacial acetic acid. The product (1.36 g) was extensively washed with $80 \%$ ethanol and dried at room temperature. For the purification of this derivative, $0.5 \mathrm{~g}$ of the sample was dissolved in $0.5 \mathrm{~L}$ of aqueous solution of $0.1 \mathrm{M} \mathrm{NaCl}$. The resulting solution was filtered and carboxymethyl chitosan was precipitated upon addition of absolute ethanol. The precipitated carboxymethyl chitosan was washed with ethanol/water mixtures of increasing ethanol content $(75 \%, 80 \%$ and $90 \%)$ and finally with absolute ethanol to desalt and dewater. Then, the purified carboxymethyl chitosan was dried at $60^{\circ} \mathrm{C}$ in an oven and finally in a vacuum desiccator at room temperature.

\section{Characterization of carboxymethyl chitosan}

Degree of substitution (DS) of prepared carboxymethyl chitosan sample was determined by $\mathrm{pH}$-metric titration?
$\mathrm{pH}$ vs. volume of sodium hydroxide titration curves were prepared as described for characterisation of chitosan ${ }^{15}$.

\section{Results and Discussion}

The fish scales of Labeo rohita were collected from local market, washed, dried and ground into small pieces. Chitin was isolated from scales of fish. Chitin molecules in the Labeo rohita fish scales are associated with collagen and minerals. It dissolved the minerals from the fish scales during demineralisation with dilute hydrochloric acid solution. Then the sample was deproteinised and the residual mass was washed thoroughly to remove the hydrolyzed protein, and dried in oven when a white solid was obtained as chitin. The percentage of yield of prepared chitin was found to be 22.36.

Chitosan was prepared from isolated chitin by deacetylation process. A white solid of chitosan was obtained after drying the deacetylated product. The prepared chitosan was insoluble in water but soluble in acidic solution below $\mathrm{pH}$ 6. Acteic acid was used for dissolving chitosan for purification process. Chitosan was precipitated at higher $\mathrm{pH}$ solution. The yield of prepared chitosan was $7.72 \%$.

To characterise chitosan prepared from fish scales of Labeo rohita, the FT-IR spectrum was recorded and compared to those of standard chitosan ${ }^{18}$. The FT-IR spectrum showed a broad absorption band at around 3483 and $3305 \mathrm{~cm}^{-1}$ indicating the intermolecular hydrogen linking formation due to the axial deformation of $\mathrm{O}-\mathrm{H}$ which appeared overlapping the bond of axial deformation of $\mathrm{N}-\mathrm{H}$. The most significant parts of chitosan spectra are those showing the amide bands at approximately 1665,1555 and $1313 \mathrm{~cm}^{-1}$. These could be assigned to the $\mathrm{C}=\mathrm{O}$ stretching the $\mathrm{N}-\mathrm{H}$ deformation in the $\mathrm{CONH}$ plane and the $\mathrm{C}-\mathrm{N}$ bond stretching plus $\mathrm{CH}_{2}$ wagging. The peaks observed at around 2932 and $2891 \mathrm{~cm}^{-1}$ were assigned to $\mathrm{sp}^{3} \mathrm{C}-\mathrm{H}$ stretching (symmetric and asymmetric). The band at $1380 \mathrm{~cm}^{-1}$ corresponded to $\mathrm{C}-\mathrm{H}$ bending and symmetric $\mathrm{CH}_{3}$ deformation, while the band at $1421 \mathrm{~cm}^{-1}$ was due to $\mathrm{CH}_{2}$ bending and $\mathrm{CH}_{3}$ deformation. The band observed at $1158 \mathrm{~cm}^{-1}$ was indicated a bridged oxygen stretching $(\mathrm{C}-\mathrm{O}-$ $\mathrm{C}$ linkage of ring). The $\mathrm{C}-\mathrm{O}$ stretching vibration bands were observed at 1116,1073 and $1025 \mathrm{~cm}^{-1}$. The characteristic peak for $\mathrm{C}-\mathrm{H}$ deformation of the $\beta$-glycosidic bond was observed at $896 \mathrm{~cm}^{-1}$. The FT-IR spectral analysis indicates the successful conversion that the isolated chitin was converted into chitosan.

Degree of deacetylation (\% DDA) is an important parameter association with the physiochemical properties of chitosan. The DDA value was determined using $\mathrm{pH}$ metric titration ${ }^{\mathbf{9}}$. In the present study, \% DDA of chitosan prepared from Labeo rohita fish scales was found to be 78.2. The degree of deacetylation of typical commercial chitosan is usually ranged between 66 and $95 \%{ }^{19}$. 
The viscosity average molecular weight of the prepared chitosan was estimated ${ }^{16}$ and found to be $1.01 \times 10^{5} \mathrm{~g} / \mathrm{mol}$. The chitosan obtained from fish scales of Tilapia (Tilapia nilotica) was reported ${ }^{13}$ to have viscosity average molecular weight of $6.88 \times 10^{4} \mathrm{~g} / \mathrm{mol}$.

Preparation and characterization of carboxymethyl chitosan from prepared chitosan

Carboxymethyl chitosan was prepared in the reaction of chitosan and chloroacetic acid in alkaline condition. The reactive sites for the carboxymethylation of chitosan are the amino and hydroxyl groups present in its chains. The carboxymethylation of chitosan occurs selectively according to the conditions used in the reaction'. The carboxymethylation reaction of chitosan generally introduces carboxymethyl groups in the hydroxyl groups bonded to the carbon atoms 3- and 6- of the glucopyranose unit. The amino group was also a reactive site and two carboxymethyl groups could be introduced. Thus, the complete characterisation of this derivative of chitosan was difficult due to its structural complexity.

The $\mathrm{pH}$ titration curves of carboxymethyl chitosan were used to determine the average value of the degree of substitution (DS) ${ }^{9}$. The DS of carboxymethyl chitosan was found to be 2.26. The titration of the sample showed the occurrence of $\mathrm{O}$ - and $\mathrm{N}$-carboxymethylation.

In the FT-IR spectrum of carboxymethyl chitosan showed adsorption bands for chitosan backbone. Despites the chitosan backbone, characteristic absorption bands for carboxymethyl chitosan were observed. The presence of strong absorption bands at around $1550-1640 \mathrm{~cm}^{-1}$ and $1400-1420 \mathrm{~cm}^{-1}$ were observed due to symmetric and asymmetric vibrations of ionized $-\mathrm{COO}^{-}$group. This indicated that carboxyl groups were grafted onto chitosan backbone when carboxymethylation reaction was occurred, and carboxymethyl chitosan was prepared successfully.

\section{Conclusion}

Chitin, isolated from fish scales of Labeo rohita, was successfully converted into chitosan by deacetylation reaction. The prepared chitosan was utilised to prepare carboxymethyl chitosan, a hydropilic biomaterial. Industries and pharmaceutical companies may develop and widely utilize many other useful products by using the chitin and chitosan from fish scales of Labeo rohita which is being thrown as waste.

1. Rinaudo, M., 2006. Chitin and chitosan: Properties and applications. Prog. Polym. Sci., 31, 603-632.

2. Muzzarelli, R. A. A., 1973. Natural Chelating Polymers. Pergamon Press, New York, p. 83.
3. Zikakis, J. P., 1984. Chitin, Chitosan and Related Enzymes. Academic Press, Orlando, p. XVII.

4. Kumar, M. N. V. R., 2000. A review of chitin and chitosan applications. React. Funct. Polym., 46, 1-27.

5. Kurita K., 2001. Controlled functionalization of the polysaccharide chitin. Prog. Polym. Sci., 26, 1921-1971.

6. Sashiwa, H. and S. Aiba, 2004. Chemically modified chitin and chitosan as biomaterials. Prog. Polym. Sci., 29, 887-908.

7. Muslim, T., M. Morimoto, H. Saimoto, and Y. Shigemasa, 2001 Synthesis of some chitosan hybrids and their metal adsorption capacities. Chitin Chitosan Res., 7, 9-18.

8. Muslim T, M. Morimoto, H. Saimoto, Y. Okamoto, S. Minami and Y. Shigemasa, 2001. Synthesis and bioactivities of poly (ethylene glycol)-chitosan hybrids. Carbohydr. Polym., 46, 323330 .

9. Abreu, F. R. and S. P. Campana-Filho, 2005. Preparation and Characterization of Carboxymethylchitosan. Polimeros, São Carlos, 15, 79-83.

10. Zhao, X., K. Kato, Y. Fukumoto and K. Nakamae, 2001 Synthesis of bioadhesive hydrogels from chitin derivatives. Int. J. Adhesion Adhesives, 21, 227-232.

11. Zaku, S. G., S. A. Emmanuel, O. C. Aguzue and S. A. Thomas, 2011. Extraction and characterization of chitin; a functional biopolymer obtained from scales of common carp fish (Cyprinus carpio 1.): A lesser known source. Afr. J. Food Sci., 5, 478-483.

12. Ikoma, T., H. Kobayashi, J. Tanaka, D. Walsh and S. Mann, 2003. Microstructure, mechanical, and biomimetic properties of fish scales from Pagrus major. J. Struct. Biol., 142, 327-333.

13. Uawonggul, N., C. Ruksakulpiwat, S. Chanthai, 2002. Study on dye-binding interactions of chitosan obtained from the fish scale of Tilapia (Tilapia nilotica). The 28th Congress on Science and Technology of Thailand, 24-26 October, Queen Sirikit National Convention Center, Bangkok, Thailand, p. 150.

14. Chandumpai, A., N. Singhpibulporn, D. Faroongsarng and P. Sornprasit, 2004. Preparation and physico-chemical characterization of chitin and chitosan from the pens of the squid species, Loligo lessoniana and Loligo formosana. Carbohydr. Polym., 58, 467-474.

15. Gutz, I. G. R., 2008. CurTipot (pH \& acid base titration curve: Analysis \& simulation) version 3.3.1 for MS Excel.

16. Huggins, M. L., 1942. The viscosity of dilute solutions of longchain molecules. IV. Dependence on concentration. J. Am. Chem. Soc., 64, 2716-2718.

17. El-hefian, E. A., A. H. Yahaya and M. Misran, 2009. Characterisation of chitosan solubilised in aqueous formic and acetic acids. Maejo Int. J. Sci. Technol., 3, 415-425.

18. Kumirska, J., M. Czerwicka, Z. Kaczyński, A. Bychowska, K. Brzozowski, J. Thöming and P. Stepnowski, 2010. Application of Spectroscopic Methods for Structural Analysis of Chitin and Chitosan. Mar. Drugs, 8, 1567-1636.

19. Agnihotri, S. A., N. N. Mallikarjuna and T. M. Aminabhav, 2004. Recent advances on chitosan-based micro- and nanoparticles in drug delivery. J. Controlled Release, 100, 5-28. 